\title{
Revisión de la implementación del SITP: la democratización y la provisionalidad*
}

\section{Review of the implementation of the SITP: democratization and provisionality}

Mauricio Alejandro Fernández Prieto**

Fecha de recepción: 20 de enero de 2018

Fecha de aprobación: 10 de abril de 2018

\section{RESUMEN}

Este artículo persigue realizar un estudio del proceso de implementación del Sistema Integrado de Transporte Público de Bogotá (SITP), visto como una política pública de movilidad, con la finalidad de encontrar la razón de sus múltiples inconvenientes a lo largo de su trayectoria. Se hará especial énfasis en los antecedentes del sistema, específicamente en dos momentos cruciales para entender el problema a fondo: uno es el proceso de democratización del sistema y el otro es la puesta en marcha del SITP Provisional, plan de emergencia creado para atender la demanda de transporte luego de los retrasos en la implementación inicial; todo bajo diferentes enfoques y modelos existentes para el análisis de las políticas públicas.

Palabras clave: implementación, política pública, movilidad, transporte, SITP, institución, democratización, provisionalidad, ciclo de las políticas públicas y top-down.

* Artículo producto del proyecto de investigación: "Revisión de la implementación del SITP: la democratización y la provisionalidad", gestionado en el marco de la investigación desde el semillero de Gobernabilidad y Políticas Públicas SEGOPP de la Facultad de Gobierno y Relaciones Internacionales FAGORI, Universidad Santo Tomás, Bogotá D. C., Colombia. DOI: http://dx.doi.org/10.15332/s1909-0528.2018.0002.02

** Profesional cum laude en Gobierno y Relaciones Internacionales, estudiante de Derecho. Universidad Santo Tomás, Bogotá D. C., Colombia. Correo electrónico: mauricio.fernandez@usantotomas.edu.co 


\begin{abstract}
This article proceeds to make a study of the implementation process of the Integrated Public Transport System of Bogota (SITP), seen as a public mobility policy, in order to find the reason of its multiple disadvantages along its trajectory. Special emphasis will be placed on the background of the system, specifically in two crucial moments to understand the problem in depth: one is the process of democratization of the system and the other is the implementation of the Interim SITP, an emergency plan created to meet the transport demand after delays in the initial implementation; all under different approaches and existing models for the analysis of public policies.
\end{abstract}

Keywords: implementation, public policy, mobility, transportation, SITP, institution, democratization, provisionality, policy cycle and top-down.

\title{
INTRODUCCIÓN
}

El Sistema Integrado de Transporte Público de Bogotá (SITP), se ha consolidado como uno de los temas imperantes en la agenda política y mediática de la capital, debido a la importancia que ha adquirido por el mero hecho de su función: transportar a los habitantes hacia su destino, posibilitando el desarrollo de sus actividades diarias y siendo el vehículo que permite materializar los múltiples derechos y libertades asociados a la movilidad. La finalidad de este artículo es determinar la fuente del retraso en la implementación de este sistema, sin perder un grado de objetividad óptimo y entendiendo el nivel de cuestionamiento constante con el que este problema público convive diariamente, es decir, desde un análisis académico.

Esta investigación de corte explicativo y correlacional, fue desarrollada aplicando un método analítico-sintético, es decir, partiendo del análisis y observación del proceso de implementación del SITP, para luego integrar todos sus componentes (actores y productos) de forma holística, a la luz de diferentes teorías sobre la implementación de las políticas públicas; todo sustentado en técnicas mixtas de recolección de datos cualitativos y cuantitativos.

Teniendo en cuenta lo anterior, se realizaron cuatro entrevistas a los actores más visibles en la ejecución del sistema. La primera de estas, a la Dirección de Implementación del SITP 
de la Empresa de Transporte del Tercer Milenio TransMilenio S. A. ${ }^{1}$; la segunda, a Marco Tulio Gutiérrez Morad² ${ }^{2}$ expresidente de la Confederación Nacional de Transporte Urbano, Conaltur ${ }^{3}$; la tercera ${ }^{4}$, a la Dirección de Transporte e Infraestructura de la Secretaría Distrital de Movilidad5; y la cuarta, a la Asociación Nacional de Propietarios de Transporte Público, Apetrans ${ }^{6}$.

\section{CONTEXTUALIZACIÓN DEL PROBLEMA DEL SITP}

El derecho a la movilidad se consagra en estos tiempos reflexivos sobre la modernidad como una necesidad fundamental. Los trayectos que debe realizar el ciudadano cada vez son más largos y numerosos dentro de los quehaceres de su cotidianidad, contrastando con los avances de la ciencia y la tecnología ${ }^{7}$, que han disminuido las distancias en tiempo y complejidad. Es necesario estar a la vanguardia del transporte para el progresivo fomento del Estado social de derecho y del cambio hacia un proyecto de desarrollo más consciente y amigable con el medio

1 Nubia Quintero fue la primera entrevistada (16 de octubre de 2015) para la realización de esta investigación. Encargada de la Dirección de Implementación del SITP, es profesional especializada de TransMilenio S. A., desempeñando labores de subgerencia técnica y de servicios en esa la entidad.

2 Marco Tulio Gutiérrez Morad fue el segundo entrevistado (20 de octubre de 2015). Abogado con amplia trayectoria en el sector público y privado en la ciudad de Bogotá, tres veces concejal y una vez representante a la Cámara por Bogotá, fue secretario de Gobierno y alcalde encargado en la alcaldía de Augusto Ramírez Ocampo, y presidente de la Confederación Nacional de Transporte Urbano Conaltur (2009-2012).

3 Entidad que agrupaba a las 64 empresas afiliadoras de transporte público colectivo (TPC) y que durante la presidencia de Marco Tulio Gutiérrez Morad, dio el paso hacia el Sistema Integrado de Transporte Público SITP.

4 La tercera entrevista tuvo lugar el 4 de noviembre de 2015 y se sostuvo frente a dos personas diferentes.

5 La primera: Carmen Rosales, directora de Transporte e Infraestructura, ingeniera de Transporte y Vías de la Universidad Pedagógica y Tecnológica de Colombia UPTC, especialista en Transporte de la Universidad Nacional de Colombia; con más de 13 ańos de experiencia en proyectos de transporte público. La segunda: Luz Dary Borrero Gómez, profesional especializada en la Dirección de Transporte e Infraestructura de la Secretaría Distrital de Movilidad de Bogotá y subordinada de Carmen Rosales, ingeniera de Transportes y Vías. Encargada de la coordinación del SITP con TransMilenio S. A., y de la implementación y el desmonte de las rutas del transporte público colectivo TPC.

6 La última entrevista se realizó el 11 de octubre de 2017 a Alfonso Pérez, presidente de Apetrans, organización de índole sindical y opositora del SITP.

7 Es importante anotar que es necesario "visibilizar el impacto que ha tenido la entrada de las nuevas tecnologías en algunos sectores del medio de transporte y el mínimo respaldo por parte del Estado en el proceso de regulación e inclusión, que no permiten brindar igualdad en todas las esferas socioeconómicas, desaprovechando los beneficios que estas puedan tener en todos los procesos" (Botero y Alegría, 2017, p. 25). 
ambiente, o como propondría Amartya Sen (1998) contrariando los viejos discursos desarrollistas basados en el desinterés por la integridad humana y la concepción vana del crecimiento económico como fuente de progreso.

El SITP es la bandera de la movilidad del nuevo milenio en la capital colombiana ${ }^{8}$, ya que propone un cambio de visión respecto al contaminante siglo $\mathrm{xx}$, que dejó un recado, el transporte público colectivo en sus siglas TPC (Urazán y Velandia, 2012); siendo este un ejemplo de lo que llamaría Roth (2002): "la ola neoliberal iniciada en los ańos 80, en pro de una sistemática reducción de las labores del Estado en favor de la delegación de tareas a privados”.

Esta política pública se pretendía lograr a partir de la eliminación de las más de 60 empresas afiliadoras que prestaban el servicio de transporte público, y el reemplazo por unas pocas empresas operadoras modernas y eficientes, cambiando el modelo de transporte y brindando un mayor control al Gobierno distrital, ya que el modelo contractual cambia de estar indefinido a ser competitivo y licitado (Acevedo, 2009).

Comienza entonces un cambio no solo en el tipo y calidad del servicio, sino en la política pública misma, teniendo en cuenta el fracaso del modelo de transporte anterior, buscando realizarse de una forma sostenible para una ciudad con tantos problemas como Bogotá. Esta sostenibilidad "se concibe como aquella capaz de satisfacer las necesidades actuales y futuras sin comprometer de manera crítica, por el riesgo que implica su extinción, los recursos necesarios para la supervivencia de las personas y el desarrollo de la población" (Duarte, 2015, p. 72).

Teniendo en cuenta dicho modelo anterior, la Secretaría Distrital de Movilidad de Bogotá se dispuso a establecer los lineamientos y principios en donde debe ahora reposar el desarrollo de la ciudad en cuanto a movilidad y transporte. Dentro de este contexto de renovación y cambio, surgieron múltiples inconvenientes en la implementación del SITP, dando origen a los retrasos conocidos en la actualidad.

Este artículo abordará el problema de la implementación, siendo constructivo sobre la puesta en marcha del SITP, que desató una ola de críticas por parte de diferentes grupos de interés,

8 En atención a lo dispuesto, es necesario hacer referencia a la necesidad de aplicar las nuevas teorías sobre el nuevo institucionalismo, como enfoque de la ciencia política, el cual se concentra en el estudio de las reglas que moldean la acción colectiva en una sociedad (Trujillo, 2018). 
además de la ciudadanía en calidad de usuaria del sistema. La fuente de estas inconformidades radica en que el SITP se encuentra estrecha e institucionalmente vinculado al sistema Transmilenio; por este motivo siempre fue visto bajo entera desconfianza, gracias al contexto sociopolítico que vivió la ciudad por culpa de los escándalos de corrupción durante la alcaldía de Samuel Moreno Rojas, donde altos cargos de instituciones distritales como el Instituto de Desarrollo Urbano IDU, el Concejo de Bogotá, la Contraloría de Bogotá e incluso la misma Alcaldía Distrital, se aliaron para contratar empresarios privados de forma fraudulenta y poder así extraer recursos públicos para beneficio propio en la construcción de la Fase III de dicho sistema (Borda, 2015; Ávila, 2011).

Finalizando esta contextualización, se procede a describir el paso a paso que se desarrollará a continuación, comenzando por los antecedentes del sistema, actores que tuvieron papel en su implementación, las disposiciones que las autoridades públicas incluyeron en su agenda al momento de ejecutar la política pública y las instancias entendiendo el SITP bajo la lógica de un modelo jerárquico; a saber: elementos fundamentales como el Plan Maestro de Movilidad, el mismo SITP en su esencia normativa, el TPC, TransMilenio, etc. Finalmente, se analizan dichos eventos desde la teoría y los autores estudiosos frente a la implementación de políticas públicas, identificando y correlacionando dos casos puntuales acontecidos durante la materialización del sistema, arrojando una visión sobre la realidad apreciable del SITP a modo de conclusión.

\section{ANTECEDENTES, ACTORES, DISPOSICIONES E INSTANCIAS EN LA IMPLEMENTACIÓN DEL SITP}

El Sistema Integrado de Transporte Público de Bogotá SITP', es el grupo de acciones para articular, vincular y operar integralmente "los diferentes modos de transporte público, las instituciones o entidades creadas para la planeación, la organización, el control del tráfico y el transporte público, así como la infraestructura requerida para la accesibilidad, circulación y el recaudo del sistema” (Decreto 319, 2006, p. 5).

9 Al respecto, es importante hacer referencia al profesor Castro (2018) para quien "Colombia ha suscrito en un tiempo récord alrededor de 14 Tratados de Libre Comercio. Ese preciso dato fáctico implica que para bien o para mal, la normatividad colombiana en muchísimos aspectos tendrá que verse reinterpretada" (p. 21). 
Las políticas públicas en el desarrollo de los objetivos del Estado moderno, funcionan "como instrumento de transformación o mantenimiento del orden social” (Roth, 2010, p. 22). El Sistema Integrado de Transporte Público ${ }^{10}$ no es otra cosa que una política pública ideada para lidiar con una problemática en materia de movilidad y transporte en Bogotá, como resultado de la actividad de diferentes autoridades que deben estar investidas tanto de poder, como de legitimidad (Meny y Thoenig, 1989/1992).

La metodología para llevar a cabo ese grupo de acciones comprende un proceso que, según la Dirección de Implementación del SITP en su entrevista, se compuso a partir de unas etapas que debían ser completadas dentro de un cronograma de actividades previamente delimitado en los contratos solemnizados entre TransMilenio S. A. y las 9 empresas operadoras ${ }^{11}$ que ganaron las licitaciones públicas organizadas por el Distrito. Estas etapas fueron prescritas por el Decreto distrital 319 de 2006, más conocido como Plan Maestro de Movilidad; esto corresponde a lo que Hill y Hupe (2002) denominarían un modelo de 'fase o etapa', cuyo objetivo principal sería la implementación desde un razonamiento lineal.

Según Roth (2002), el análisis de políticas públicas tiene como principal propósito identificar los actores y elementos que intervienen en su fabricación. Para Parsons (1995/2007) existen distintos enfoques para el análisis que se plantea desde André-Noël Roth. Dentro de la gama aportada por Wayne Parsons en su libro y concordando con Hill y Hupe (2002), se encuentra el enfoque por etapas: este se preocupa por estudiar las políticas públicas buscando entenderlas dentro de una serie de fases, cada una con una finalidad en específico, de tal forma que son conducentes entre sí y plantean una estructura lógica y coordinada de comprensión y análisis (Parsons, 1995/2007).

Entendiendo conceptualmente el SITP, se evidencian distintas operaciones delimitadas que son coherentes entre sí, y que son identificables dentro de lo que se conoce como el "policy cycle” o ciclo de las políticas públicas, concepto inmerso dentro del enfoque sugerido por Parsons. Este proceso abarca diferentes fases, y aunque hay diferentes concepciones acerca

10 De conformidad con González (2017): "El transporte público y el tráfico están asociados a los factores económicos y técnicos que condicionan los desplazamientos de las personas, en tanto que la movilidad se centra en los individuos y su entorno. Aunque son dimensiones diferentes, es claro que una adecuada planificación del transporte tiene una positiva incidencia en la movilidad" (p. 150).

11 "Empresa que administra, programa, mantiene y repara de manera conjunta la totalidad del parque automotor con que cuenta” (Decreto 319, 2006, art. 3). 
de cómo se compone este ciclo: por ejemplo Simon en 1947, Lasswell en 1956, Mack en 1971, Rose en 1973, Jenkins en 1978 y Hogwood y Gunn en 1984 (Parsons, 1995/2007); la versión más conocida es la propuesta por Charles O. Jones en 1970, que consta de 9 pasos (percepción, definición, organización, representación, formulación, legitimación, aplicación, reacción y evaluación).

La propuesta de Jones fue popularizada por James E. Anderson, quien logró sintetizar dicho modelo en 1975 estableciendo cinco elementos puntuales, que son: la identificación del o de los problemas, la formulación de posibles soluciones, la toma de decisiones, la ejecución de lo planeado y por último, la evaluación de lo logrado (Anderson, 2015).

En materia de identificación (primera fase), la problemática a solventar era poder reemplazar la anterior modalidad de transporte, el denominado transporte público colectivo en sus siglas TPC, un sistema heredado del siglo pasado, consolidado en pleno auge del neoliberalismo a inicios de los años noventa (Asprilla y Rey, 2012), pues su naturaleza es $100 \%$ privada. El Estado decidió en ese entonces delegar esa función al mercado, al actor privado, situación que no resultó para nada bien, pues el margen de riesgo y accidentalidad, junto con la falta de control en cuanto a contaminación por combustibles fósiles, la inseguridad y la baja calidad del servicio en general, hacían de esta modalidad de transporte público un problema latente para la ciudad (Urazán y Velandia, 2012); como reafirmaría la Dirección de Transporte e Infraestructura de la Secretaría de Movilidad, el TPC no era querido por el ciudadano, a diferencia de otros sistemas como el metro de Medellín.

El TPC "son los buses, busetas y microbuses que no son parte del sistema TransMilenio" (Ardila, 2005, p. 57), y que según el entrevistado Marco Tulio Gutiérrez Morad, son un sistema compuesto por tres segmentos: unas cooperativas, unos conductores y por último, el usuario, quien llevaba consigo la peor parte. La Figura 1 clarifica la composición del transporte público colectivo. 
Cooperativas: tenían las facultades legales para administrar las rutas sin regulación eficiente, por este motivo se presentaron casos de corrupción.

Buseteros o transportadores: podían ser propietarios de vehículos o empleados, al existir permisividad por parte de la administración el servicio era de mala calidad.

Usuarios: se veían sometidos a los abusos de las cooperativas, inseguridad en la prestación del servicio y negligencia de las autoridades competentes.

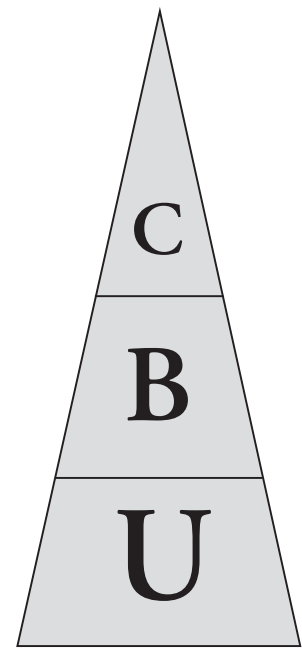

Figura 1. Representación del sistema jerárquico del transporte público colectivo (TPC)

Fuente: elaboración propia con base en: Ardila (2005), Asprilla y Rey (2012), Urazán y Velandia (2012) y entrevistas realizadas para esta investigación.

Dicho sistema de cooperativas "evidenció un notable deterioro de la calidad y prestación del servicio, mayor congestión (trancones), sobreoferta, 'guerra del centavo'12, accidentalidad, contaminación ambiental, caos en la prestación del servicio público y una débil institucionalidad" (Asprilla y Rey, 2012, pp. 28-29), enfatizando en que dicha debilidad se debe entender como un entramado de procesos corruptos, existentes gracias a la complicidad de empresas cooperativas que solo buscaban maximizar sus ganancias sin importar la situación tan deplorable de la ciudad en cuanto a transporte, y los funcionarios corruptos de las organizaciones estatales, incluso de altas dignidades de la rama judicial (Ardila, 2005).

12 La guerra del centavo es el nombre popularmente dado a la irresponsable actividad entre conductores del sistema TPC por recoger el mayor número de pasajeros posible, pues competían en carreras con quienes tenían la misma o similar ruta y podían llegar a manejar de forma no apropiada el vehículo y violentar infinidad de normas de tránsito para aumentar su margen diario de ganancias (ver Lleras, 2005 y Urazán y Velandia, 2012). 
Cabe resaltar que el primer proyecto ejecutado para solventar este problema no fue el SITP, sino el sistema Transmilenio ${ }^{13}$, que en sus inicios tuvo una acogida positiva por parte de los usuarios del transporte público en Bogotá, incluso durante sus primeros años se consideró como una de las soluciones más factibles respecto a calidad-precio que podía darse a futuro en la capital (Lleras, 2005).

Entre los antecedentes que dieron origen a TransMilenio S. A. ${ }^{14}$, empresa de economía mixta (participan en ella capitales públicos y privados) y que además está adscrita a la Secretaría de Movilidad, figura desde el intento de un Sistema Integrado de Transporte Masivo siтm en el año de 1995, obra de acuerdos entre el Gobierno nacional (expresidente Ernesto Samper) y el Gobierno local (exalcalde Antanas Mockus); hasta la realización de estudios por parte de la Agencia de Cooperación Internacional de Japón ${ }^{15}$, JICA por sus siglas en inglés, que dieron origen al Plan Maestro de Transporte Urbano ${ }^{16}$, primer precedente del Sistema de Transporte Masivo Transmilenio TM en 1998 (Asprilla y Rey, 2012).

En vista de que Transmilenio no fue suficiente para mitigar las persistentes consecuencias (que se verán más adelante) del TPC, que a la fecha de publicación de este artículo se siguen manifestando en el diario vivir de los bogotanos; la formulación de alternativas de solución y su correspondiente toma de decisión (segunda y tercera fase del ciclo de las políticas públicas), comprendió como tal, la propuesta del SITP para erradicar el problema del transporte público en la ciudad.

13 La modalidad Transmilenio se basa en un sistema de buses articulados (rojos), biarticulados (rojos con amarillo) o duales (rojos con gris) que transitan por troncales (vías primarias de alta prelación [Art. 105 Código Nacional de Tránsito Terrestre-Ley 769 de 2002] exclusivas para la movilización del transporte público y masivo), y que realiza paradas en estaciones y portales. Para más información ver: http://www.sitp.gov.co.

14 Para evitar una confusión de términos, hay que esclarecer que 'Transmilenio' o 'transmilenio', hace referencia a la modalidad de transporte mencionada en el anterior pie de página, mientras que 'TransMilenio S. A.' es una empresa de economía mixta obedeciendo a la naturaleza de estas instituciones según la Ley 489 de 1998.

15 Lo dispuesto, está en perfecta correspondencia con el hecho de que "América Latina no ha sido ajena al fenómeno de la globalización y a las necesidades sociales que esta acarrea, de tal forma que, al igual que los principales países del mundo, ha tratado de adaptarse a medida que se han presentado dichas necesidades; claro está, de una forma más lenta que los demás, debido a sus condiciones económicas, sociales y políticas" (Llano, Rengifo y Rojas, 2018, p. 99).

16 No se debe confundir el Plan Maestro de Transporte Urbano, un documento de política pública gestado durante la alcaldía de Antanas Mockus (1995-1997), con el Plan Maestro de Movilidad PMM, actual documento guía de política pública originado durante la alcaldía de Luis Eduardo Garzón (2004-2008). 
El sistema de buses articulados Transmilenio siguió operando y se redujo a una de varias nuevas modalidades de transporte público que en la actualidad se encuentran integradas, y la empresa TransMilenio S. A. quedó delegada como la institución encargada del SITP, específicamente "como ente gestor del transporte masivo, tiene la responsabilidad de la integración, evaluación y seguimiento de la operación del SITP” (Decreto 319, 2006, art. 15); todo bajo la tutela de la Secretaría de Movilidad del Distrito Capital (antes Secretaría de Tránsito y Transporte).

Luego de haber abarcado el problema, lo que procedieron a realizar las autoridades públicas encargadas del asunto hacia el año 2006, fue dar origen al Plan Maestro de Movilidad, Decreto de orden distrital, emitido por el exalcalde Luis Eduardo Garzón el 15 de agosto de 2006, que busca dar trámite a políticas, programas, planes, proyectos, etc., que logren una movilidad ideal, "institucionalmente coordinada, y financiera y económicamente sostenible para Bogotá y para la región” (Decreto 319, 2006, art. 8).

Teniendo en cuenta los antecedentes anteriores, el SITP es el resultado de muchos intentos de querer integrar los modos de transporte público de Bogotá, inicialmente como un sistema bimodal, donde solo convivirían Transmilenio y el TPC (Lleras, 2005), y luego, en palabras de la Dirección de Transporte e Infraestructura: en un esquema ampliado que no solo integra más modalidades existentes, sino que su finalidad es acoplar cualquier otra que a futuro se llegue a planificar, por ejemplo el metro que actualmente es tema de discusión en la agenda pública de la ciudad.

Comprendiendo el problema del SITP, según Hill y Hupe (2002) concordando con lo dicho por Wayne Parsons, el policy cycle es una herramienta que actúa como facilitadora del análisis de la política. La tarea de contextualizar los antecedentes que obedecen a las relaciones entre autoridades responsables junto con sus decisiones, para Laurence J. O’Toole (2000) en el campo de la investigación en implementación de políticas, funciona para "incorporar una comprensión más completa de los múltiples niveles de acción y tipos de variables que se puede esperar que influyan en el rendimiento" (p. 276). En resumidas cuentas, es ahora más sencillo identificar la importancia de la fase cuatro de ejecución o implementación, cuya transcendencia en materia de análisis de políticas públicas, sin menospreciar las etapas de identificación, formulación y decisión, es el principal objetivo de este artículo.

El modelo top-down (de arriba hacia abajo), que fue desarrollado por Paul Sabatier, consiste en el análisis de las decisiones que toman los implementadores de la política (Revuelta, 2007). 
Wayne Parsons advierte el razonamiento secuencial en las políticas públicas que siempre "tiende naturalmente a prestarse al enfoque 'gerencialista' y 'de arriba hacia abajo' del proceso de las políticas públicas, y a mirar el papel del análisis de las mismas como una especie de recurso elitista" (Parsons, 1995/2007, p. 115).

El top-down plantea una serie de pasos específicos esperando que de forma precisa se logren los resultados prescritos inicialmente, bajo la creencia en que la autoridad posee pleno control de las relaciones institucionales bajo las que se da la implementación de las políticas (Elmore, 1978), coincidiendo con Meny y Thoenig (1989/1992) cuando hablan de la primacía jerárquica de la autoridad, donde "la administración, que es la espina dorsal de la implementación, se parece a una pirámide jerarquizada y centralizada” (p. 159), lo que sugiere una lógica lineal.

Al ser el SITP una política pública ${ }^{17}$ construida secuencialmente, el actor predominante en el proceso de elaboración de la política es la autoridad. La Secretaría Distrital de Movilidad y TransMilenio S. A. se valieron de sus capacidades legales y administrativas para implementar el sistema, buscando atender un problema social, sin olvidar a las empresas operadoras mencionadas anteriormente, nuevos actores que entrarían a ejecutar sus respectivos contratos y que serán tenidas en cuenta más adelante.

Buscando establecer cronológicamente esta corriente de implementación, Narendra Raj Paudel (2009) retoma el modelo top-down y lo sitúa en la segunda generación de investigación de la implementación en políticas públicas (ańos 80 y 90), que se caracterizó grosso modo en el análisis de las relaciones entre la política pública y la realidad de esta en la práctica, diferenciándose de la primera generación (años 70 a 80), donde imperaba más un enfoque prescriptivo y teórico. También resaltó la importancia de cambios en variables como los períodos de tiempo y los resultados a nivel local que obtenían las políticas al implementarse.

$17 \mathrm{Al}$ respecto, es importante atendiendo la razón de ser del servicio público de transporte, no olvidar que "El modo de inserción de gran parte de los países de América Latina, y particularmente los andinos, en la globalización ha sido precario, incierto y frágil. Entidades locales que podrían aparecer momentáneamente como ganadoras por contar con ventajas vinculadas a mercados, precios o infraestructura, no tienen un estatuto consolidado y, como consecuencia de ello, pueden rápidamente desplomarse en las cadenas de producción o circulación transnacional" (Blanco, 2017, p. 67) 
Teniendo en cuenta la primera generación de estudios, implementar para Pressman y Wildavsky (1984) es llevar a cabo, cumplir, producir o completar una tarea determinada. Siendo esta definición un tanto abstracta, Donald Van Meter y Clark Van Horn (1975) plantean que:

La implementación de políticas abarca aquellas acciones de individuos (o grupos) públicos y privados dirigidas al logro de los objetivos establecidos en las decisiones políticas anteriores. Esto incluye esfuerzos únicos para transformar las decisiones en términos operativos, así como esfuerzos continuos para lograr los grandes y pequeños cambios impuestos por las decisiones políticas (p. 447).

Paul Sabatier y Daniel Mazmanian (1983) añaden un elemento adicional, consideran que "la implementación de una política pública es llevar a cabo una decisión básica de esa política, la cual usualmente está incorporada en un estatuto, esta también puede tomar forma y ser una orden ejecutiva o decisiones de una corte" (pp. 20-21). Van Meter y Van Horn también plantean este factor (estatuto) como una ley previamente promulgada o cualquier tipo de norma sin hacer mayor distinción (Revuelta, 2007).

Teniendo en cuenta el cambio en los estudios de implementación, la segunda generación, de la que hacen parte Yves Meny y Jean-Claude Thoenig, concibe ese estatuto más como un "marco normativo de intenciones" cuya existencia se justifica en materializar en la realidad lo planeado, teniendo en cuenta tres principios: 1) la ya mencionada primacía jerárquica de la autoridad, en donde se resalta la necesidad del concepto de burocracia aportado por Max Weber, 2) la distinción entre lo político y lo administrativo, donde este segundo debe tener prelación sobre el primero para una mayor objetividad y, 3) la eficiencia, que aboga por optimizar todos los recursos disponibles al momento de gestionar para obtener resultados satisfactorios (Meny y Thoenig, 1989/1992, pp. 158-159).

El Plan Maestro de Movilidad (como 'estatuto') bajo la lógica lineal propuesta, se convierte en un elemento vital para la implementación del SITP, que como se ha enfatizado, corresponde a una gestión de tipo top-down. Es ahora necesario profundizar respecto a la autoridad encargada de la fase de ejecución, pues existen diferencias entre los actores señalados con anterioridad, a saber: La Secretaría de Movilidad y TransMilenio S. A.

Meny y Thoenig (1989/1992), citando a Maytnz (1980), invitan a establecer una distinción entre una estructura de ejecución y un proceso de ejecución, ambas importantes desde el 
punto de vista de la autoridad que decide implementar las políticas públicas; esto se relaciona con lo expuesto por Michael Hill y Peter Hupe (2002), quienes argumentan que el proceso de implementación comienza con dos tipos de actores: el primero, es el funcionario político, quien proporciona los objetivos, las bases y los principios bajo los cuales debe regir la política a ejecutar; el otro actor es el administrador, encargado de desarrollar los instrumentos más apropiados para materializar lo dispuesto por el funcionario político.

La estructura de ejecución es competencia del funcionario político, que es representado por la Secretaría Distrital de Movilidad de Bogotá, la cual dispuso el Plan Maestro de Movilidad como estatuto o documento guía que aglomera los elementos sustanciales mencionados anteriormente; y el proceso de ejecución es puesto en marcha por el ya mencionado gestor o administrador, que en el caso puntual del SITP, es TransMilenio S. A., encargado de la parte procedimental y práctica de la implementación.

Abordando el concepto de burocracia resaltado por Meny y Thoenig, para Joan Subirats (1992) toma vital importancia en la fase de implementación, ya que para este autor, la función de este proceso que tiende a ser repetitivo y lento es precisamente el de ejecutar lo dispuesto por los decisores políticos; cabe resaltar que para Subirats la fase de ejecución parte desde algo incierto, para él los estatutos de los que hablan Sabatier y Mazmanian en 1983, buscan siempre ser ambiguos con el motivo de otorgar autonomía a los ejecutores en materia práctica, que en este caso viene siendo la empresa TransMilenio S. A. Esta ambigüedad puede en muchos casos ser tropiezo para el proceso de implementación secuencial.

Ejemplo de lo anterior es la afirmación de la directora de Implementación del SITP entrevistada, pues una herramienta para evitar el incumplimiento de los contratos fue incluir en las cláusulas un indicativo que permitía posponer los plazos de implementación, teniendo en cuenta las adversidades que se pudiesen presentar en el momento de materializarse el sistema. Esto lo que provocó fue posponer progresivamente las entregas planificadas y generar retrasos que, a la luz del contrato, no tenían mayor relevancia, pero sí representaban un problema como obligación con ese principio de eficiencia que exponen Meny y Thoenig.

Aguilar y Lima (2009) argumentan que no existe un solo implementador, sino multiplicidad de actores que determinan el curso de la política pública; esto constituye una de las mayores dificultades al momento de iniciar la fase de ejecución, esta es la coordinación entre la institución principal y las demás subdirecciones adscritas e involucradas en el proceso de imple- 
mentación de políticas (Sabatier y Mazmanian, 1980). Para Meny y Thoenig (1989/1992), es entonces imposible que el encargado de ejecutar la política pública actúe por su cuenta, pues necesita de otros actores o grupos para dar trámite a la encomienda realizada por el funcionario político.

Lo expuesto anteriormente, es la síntesis de los problemas que se abordarán más adelante y que corresponden a desentendimientos entre los actores protagonistas en el SITP. En la entrevista realizada a TransMilenio S. A., la Dirección de Implementación enfatizó en esta situación, pues para la entidad una de las mayores dificultades en el proceso de ejecución, fue la coordinación institucional con el ente delimitador (Secretaría de Movilidad) y más aún con los operadores privados con los que persisten problemas.

Para poder apreciar estas aproximaciones teóricas sobre la implementación de políticas públicas en la práctica, la segunda parte de este artículo expondrá dos ejemplos que evidencian errores por parte de las autoridades, bajo la lógica del modelo secuencial de ejecución del Sistema Integrado de Transporte Público, SITP.

\section{CASOS DE ESTUdio: LA DEMOCRATIZACIÓN Y LA PROVISIONALIDAD DEL SITP}

La implementación del SITP al constatarse en la teoría, deja ver una serie de elementos y conceptos que evidencian una realidad muy distinta a lo prescrito bajo la lógica del modelo secuencial explicado anteriormente. Sin embargo, es necesario analizar dos situaciones producto de la gestión realizada por las autoridades competentes, logrando justificar por medio de la figura argumentativa del ejemplo, los fallos en la materialización del sistema.

Recordando que los proponentes ganadores de las licitaciones realizadas por TransMilenio S. A. son actores de naturaleza privada, más allá de vínculos contractuales con el Estado, hicieron parte de un modelo de política pública ideado en conjunto entre el sector público y el sector privado, en donde "el Estado debe transferir progresivamente a estas organizaciones la responsabilidad de facilitar y llevar adelante los servicios sociales" (Stahl, 1994, p. 12); impera la necesidad de establecer un origen causal de los retrasos del sistema por parte de los actores privados, lo que se realizará a partir de dos situaciones durante el proceso de implementación del SITP.

66 
Hubo dos empresas que presentaron problemas y no implementaron su parte adjudicada, a saber: Egobús S. A. S. ${ }^{18}$ y Coobús S. A. S., teniendo contratado más del $42 \%$ del sistema no cumplieron con lo pactado y fueron intervenidas por la Superintendencia de Puertos y Transporte, con quien a pesar de lograr acordar planes de salvamento (Superintendencia de Puertos y Transporte, s. f.), les fue declarada la caducidad del contrato por parte de TransMilenio S. A. Además, por orden de la misma Superintendencia, esta decretó la apertura de la liquidación judicial de dichas personas jurídicas (Superintendencia de Sociedades, 2016).

Comprendiendo lo anterior, el sistema actualmente está conformado tan solo por 7 operadoras de las 9 iniciales, las zonas no implementadas (adjudicadas a Coobús y Egobús) para evitar complicaciones mayores a las ya existentes, mantienen un esquema de transporte público en su gran mayoría, operado por lo que se conoce como SITP Provisional.

\section{Caso de estudio $\mathrm{N}^{\circ}$ 1: la democratización del SITP}

Para O’Donnell y Schmitter (1988), la democratización como concepto es un período de transición que se da de un régimen anterior, cuya principal característica es la ausencia total o parcial de participación de la ciudadanía y de grupos históricamente ajenos al poder político, hacia uno nuevo donde se realiza lo que Jaime F. Cárdenas (2005) describe como un "proceso de devolución de soberanía” (p. 31) a dichos actores y colectividades.

Teniendo en cuenta ese estado anterior de limitado margen de participación, buena parte de los ganadores de las licitaciones públicas del SITP son grandes y prestigiosas empresas que ya tenían troncales y portales de Transmilenio adjudicados desde la creación de TransMilenio S. A. (Dinero, 2010); el Distrito buscando ser inclusivo con los transportadores tradicionales del TPC que saldrían del negocio, dispuso en el Plan Maestro de Movilidad un concepto que buscó proteger a estos empresarios y evitar conflictos en el futuro.

La 'democratización' del SITP fue uno de los principios a consideración de los entrevistados, que más tuvo influencia en la constitución del sistema. Consiste en la implementación de la política pública sin discriminar a los miembros del TPC, es decir, "propiciar, en los nuevos con-

18 S. A. S. es una sigla que significa 'sociedad por acciones simplificada' (para más información consultar la Ley 1258 de 2008). 
tratos de operación, la participación del mayor número posible de propietarios actuales de vehículos de transporte público colectivo matriculados en Bogotá" (Decreto 309, 2009, art. 15).

El SITP en la actualidad no se puede considerar democratizado, pues la mayoría de los miembros del transporte público colectivo (TPC) han salido del proceso de implementación. Para contextualizar, el SITP estuvo conformado por 9 empresas ganadoras de las licitaciones de las 13 zonas en que fue dividida la ciudad. La Tabla 1 evidencia cómo fueron adjudicadas las zonas según los concesionarios ${ }^{19}$ de buses ganadores y el porcentaje de avance que han logrado.

Tabla 1. Cuadro de concesionarios operadores de buses y zonas licitadas adjudicadas

\begin{tabular}{|l|l|l|}
\hline \multicolumn{1}{|c|}{ Empresa operadora } & \multicolumn{1}{|c|}{ Zona adjudicada } & \multicolumn{1}{c|}{ Avance* } \\
\hline TranZit S. A. S. & Zona 12 Usme & $78 \%$ \\
\hline GMOVIL S. A. S. & Zona 5 Engativá & $100 \%$ \\
\hline Masivo Capital S. A. S. & Zona 8 Kennedy y Zona 2 Suba Oriental & $\begin{array}{l}91 \% \text { y } 100 \% \\
\text { respectivamente }\end{array}$ \\
\hline ETIB S. A. S. & Zona 9 Bosa & $97 \%$ \\
\hline Este es Mi Bus S. A. S. & $\begin{array}{l}\text { Zona 4 Calle 80 y Zona 7 Tintal - Zona } \\
\text { Franca. }\end{array}$ & $\begin{array}{l}82 \% \text { y } 84 \% \\
\text { respectivamente }\end{array}$ \\
\hline Suma S. A. S. & Zona 11 Ciudad Bolívar & $74 \%$ \\
\hline${ }^{*}$ Egobús S. A. S. ${ }^{* *}$ & Zona 10 Perdomo y Zona 3 Suba Centro & No aplica \\
\hline *Coobús S. A. S. ${ }^{* * *}$ & Zona 6 Fontibón & No aplica \\
\hline Consorcio Express S. A. S. & Zona 1 Usaquén y Zona 13 San Cristóbal & $\begin{array}{l}85 \% \text { y } 98 \% \\
\text { respectivamente }\end{array}$ \\
\hline
\end{tabular}

Fuente: elaboración propia con base en la información suministrada por el portal web oficial del SITP: http://www. sitp.gov.co/Publicaciones/el_sistema/operadores_del_sistema y derecho de petición contestado al autor por TransMilenio S. A. con radicado 2017ER7478.

* Avance a fecha del 25 de abril de 2017. Información obtenida de derecho de petición contestado al autor por TransMilenio S. A. con radicado 2017ER7478.

** Egobús S. A. S. (sociedad por acciones simplificada) actualmente no hace parte del SITP.

*** Coobús S. A. S. (sociedad por acciones simplificada) actualmente no hace parte del SITP.

19 Los concesionarios son las mismas empresas ganadoras de las licitaciones, este nombre tiene origen operativo, es decir, son concesionarios de buses los que implementan las rutas, siendo un concepto meramente administrativo. 
Si en algo coincidieron las instituciones responsables del SITP entrevistadas para esta investigación (TransMilenio S. A. y la Secretaría Distrital de Movilidad) con el gremio de transportadores Conaltur y la asociación Apetrans, es que la culpa de por qué Egobús S. A. S. y Coobús S. A. S., empresas conformadas por los viejos empresarios tradicionales no lograron implementar su parte del sistema, se encuentra en la naturaleza misma de estas, más específicamente en su composición administrativa.

Cuando se habla de la naturaleza de las empresas, nos referimos al modelo económico de estas, que según el entrevistado Gutiérrez Morad, expresidente de Conaltur, coincidiendo con lo argumentado por Asprilla (2005), Lleras (2005) y Urazán y Velandia (2012), proviene del sector cooperativo tradicional colombiano; las cooperativas en Colombia fueron siempre de ahorro y crédito, pero nunca de inversión, sosteniendo de esta forma el modelo TPC anterior.

Los viejos transportadores estaban entonces acostumbrados a un modelo económico muy distinto al que TransMilenio S. A. les ofreció en la democratización del sistema, pues este se compone de principios como la financiación y la inversión de capitales; su mentalidad empresarial comprendía ingresos con el objeto de que fueran empleados para ahorrar en la cooperativa a la que estaban afiliados, obtener créditos menores y mantener una estabilidad económica sin una mayor proyección de crecimiento.

El que se les propusiera invertir a gran escala en un nuevo negocio, produjo el fracaso empresarial de Coobús y Egobús. Estas 2 empresas, según el expresidente de Conaltur, perdieron los avales financieros de los bancos debido a disputas internas en los concesionarios y terminaron incumpliendo sus respectivos contratos. El entrevistado, a pesar de reconocer esta falta por parte del gremio que representa, sostiene que las autoridades encargadas de implementar no acompańaron a los viejos transportadores y los dejaron a la deriva en este proceso de cambio de modelo de negocio, situación que confirmaría Apetrans en su entrevista, pero extendido sobre las disputas internas, pues atribuyen culpa al Distrito por este hecho.

Las autoridades que generalmente se guían por modelos secuenciales top-down, tienden a descuidar lo que ocurre en la realidad, dando mayor relevancia a las prescripciones que contienen los estatutos e ignoran lo que los implementadores de menor nivel jerárquico pueden llegar a provocar desarrollando sus respectivas tareas (Paudel, 2009). 
La visión por parte de los implementadores que están en la cima de la jerarquía de decisión, es negativa respecto a los que se encuentran debajo de ellos, en síntesis, estos argumentan que la culpa es exclusiva de los contratistas. Esta conducta resulta bastante recurrente en la lógica secuencial top-down, ya que "los modelos de arriba hacia abajo, sin embargo, ven a los actores locales como impedimentos para una implementación exitosa, agentes cuya conducta de evasión necesita ser controlada” (Matland, 1995, p. 148).

Esta perspectiva que manifiestan los decisores sobre los implementadores de menor nivel, tiene origen en un principio del modelo secuencial: la teoría de la elección racional, que supone "que quienes intervienen en la implementación de las políticas públicas (policy-makers) son actores racionales" (Peters, 1992 en Estévez y Esper, 2005, p. 74), por lo tanto, todas sus decisiones deben presumirse como correctas.

Sin embargo, esta creencia es bastante cuestionada. Charles E. Lindblom (1959) sostiene que la elección racional en estas circunstancias carece de validez en la práctica, pues "supone capacidades intelectuales y fuentes de información que los hombres simplemente no poseen" (p. 80), desvirtuando la existencia de soluciones perfectas que se ven condicionadas por la misma naturaleza finita del ser humano, este es el principio de lo que se conoce como la teoría de la racionalidad limitada (Estévez y Esper, 2005).

La democratización del SITP es un excelente exponente de esta falencia del modelo secuencial de implementación, las políticas públicas que se gobiernan bajo el top-down siempre tienden a fracasar por su afán de concentrarse en la prescripción teórica, más no en la práctica (Elmore, 1979 en Paudel, 2009).

\section{Caso de estudio $\mathrm{N}^{\circ}$ 2: la provisionalidad del SITP}

El SITP Provisional fue la última de cuatro estrategias (contingencia, modificación plan de implementación, rutas compartidas y cobertura con rutas provisionales ${ }^{20}$ como respuesta institucional a los problemas y retrasos en la implementación de distintas rutas de buses, por parte de las 2 empresas operadoras disueltas y liquidadas, que fueron incluidas en el sistema

20 Información obtenida de derecho de petición contestado al autor por TransMilenio S. A. con radicado 2017 ER7478. 
gracias al principio de democratización inmerso en el Plan Maestro de Movilidad, adjudicándoseles el para nada irrelevante $42 \%$ de la operación total del sistema.

La primera estrategia, "contingencia”, tuvo por objetivo destinar vehículos de otros concesionarios de forma temporal para evitar la suspensión de las escasas 15 rutas que alcanzaron a implementar Coobús S. A. S. y Egobús S. A. S. en su corta duración como contratistas. La segunda estrategia, "modificación plan de implementación”, consistió en aplazar algunas rutas de los planes de implementación de los otros concesionarios con la finalidad de destinar esos buses para estabilizar la primera estrategia. La tercera estrategia, "rutas compartidas", combinó rutas que compartían los concesionarios liquidados para usar menos buses y las puso a funcionar con parte de la flota de las empresas operadoras activas. En vista de que estas empresas se quejaron por no poder implementar sus partes por estar ayudando a TransMilenio S. A. con este problema, surgió la idea de la cuarta estrategia.

Comprendiendo la magnitud del problema, es decir, el que no se hubiera implementado nada concreto de ese $42 \%$, habiendo transcurrido casi cuatro ańos desde su inicio (20112015), la Secretaría de Movilidad, por medio de la Resolución 518 (2015), no tuvo otra opción que poner en funcionamiento la cuarta estrategia, "cobertura con rutas provisionales", que otorgó permisos especiales y transitorios a los 'buseteros' tradicionales del TPC, con la condición de que deberían ser administrados por TransMilenio S. A., atendiendo temporalmente la demanda de buses y rutas de los usuarios.

La decisión por parte de la administración de retornar a los buses tradicionales, así fuese de forma transitoria, no fue bien recibida por los bogotanos en general, teniendo en cuenta la baja popularidad del transporte público colectivo (TPC) entre los usuarios según el entrevistado Gutiérrez Morad; en la actualidad, luego de más de dos años de haberse ejecutado aún persiste la provisionalidad del sistema, a pesar de que, como se puede constatar en el artículo $1^{\circ}$ de la norma, inicialmente fue una estrategia aprobada por solo 2 meses (Resolución 518 de 2015).

La Tabla 2 evidencia la situación que aún en pleno 2017 se seguía presentando con la movilidad y el transporte público en Bogotá, más de 5000 buses tradicionales del TPC que, con calcomanías del logo del SITP y un número de identificación, volvieron a operar para mitigar la demanda de transporte de forma provisional (Alcaldía Mayor de Bogotá, 2015). 
Tabla 2. Cuadro comparativo entre el SITP Provisional y el SITP

\begin{tabular}{|l|l|}
\hline \multicolumn{1}{|c|}{ SITP Provisional } & \multicolumn{1}{c|}{ SITP } \\
\hline Desventaja: recaudo de pasajes en efectivo & $\begin{array}{l}\text { Ventaja: recaudo de pasajes por medios } \\
\text { electrónicos }\end{array}$ \\
\hline Desventaja: 'guerra del centavo' & $\begin{array}{l}\text { Ventaja: salario por medio de contrato que } \\
\text { extingue la competencia entre conductores }\end{array}$ \\
\hline $\begin{array}{l}\text { Desventaja: contaminación ambiental por } \\
\text { negligencia de las autoridades responsables }\end{array}$ & $\begin{array}{l}\text { Ventaja: responsabilidad ambiental inmersa en el } \\
\text { Plan Maestro de Movilidad }\end{array}$ \\
\hline $\begin{array}{l}\text { Desventaja: generación de tráfico vehicular por } \\
\text { la no existencia de un sistema de paraderos }\end{array}$ & $\begin{array}{l}\text { Ventaja: existencia de un sistema de paraderos } \\
\text { con indicadores de las rutas que se pueden } \\
\text { abordar }\end{array}$ \\
\hline
\end{tabular}

Fuente: elaboración propia con base en las entrevistas realizadas a la Secretaría Distrital de Movilidad, a TransMilenio S. A. y al expresidente del gremio de transportadores urbanos, Conaltur.

La implementación es el punto de contraste de lo inicialmente propuesto y lo realmente constituido como política pública en la realidad, es decir, lo materializado por los entres gestores o administradores, ya que las repercusiones o consecuencias en la parte sustancial de la política pueden constituir algo totalmente diferente a lo estipulado en sus inicios (Anderson, 1975 en Hill y Hupe, 2002).

La provisionalidad del SITP es clara evidencia del fracaso en el modelo lineal, por medio del cual las autoridades decidieron implementar la política pública sin entender a los actores que, a pesar de no ostentar la misma jerarquía burocrática, no son menos importantes. La principal crítica del modelo top-down tiene origen también en la segunda generación, o tercera para otros (Peńa, 2011), de los estudios de implementación de políticas, esta es desde el modelo bottom-up (de abajo hacia arriba), que implica una multiplicidad de actores que intervienen directa o indirectamente en el proceso de implementación de las políticas públicas, acentuando en la variedad de canales que unen a estos actores y la posibilidad de utilizar estas conexiones para negociar sobre sus intereses (Morata, 1990).

En el bottom-up el concepto de burocracia desarrollado en la primera parte del artículo cambia considerablemente, pues supone que no existe una sola burocracia naturalmente institucional, sino una segunda, 'de la calle': "se considera que los burócratas de la calle tienen una 
mejor comprensión de lo que necesitan los clientes, ya que son ellos quienes tienen contacto directo con el público" (Paudel, 2009, p. 41).

Según Michael Lipsky (2010), los burócratas de la calle al tener ese trato especial con la ciudadanía, además de su relativamente alto grado de discrecionalidad en sus actuaciones respecto de sus intereses y su autonomía de la autoridad, poseen capacidades que simplemente no se pueden pretender ignorar en el proceso de elaboración de las políticas públicas por parte de las autoridades, más aún en la etapa de implementación.

Para los defensores de la construcción de políticas públicas desde la autoridad, la ejecución se debe dar del centro hacia la periferia (Meny y Thoenig, 1989/1992), mientras que otros consideran que "no es solo decidir e implementar; es conocer cuál es el papel que está desarrollando cada agente que interviene" (Calderón, 2016, p. 75).

Bajo este entendido, el proceso de ejecución se materializa en dos niveles: uno macro, donde las autoridades llevan a cabo una política, y otro micro, donde actores de menor jerarquía reaccionan a las decisiones del nivel macro (Berman, 1978 en Paudel, 2009).

Paul Berman (1978), al analizar estos conceptos de macro y microimplementación en su estudio realizado en Estados Unidos, concluye que:

El Gobierno federal generalmente tiene capacidad limitada para influir en el comportamiento de los ejecutores locales, que tienen poder efectivo en el sistema de políticas [...] la microimplementación no puede ser efectiva a menos que las organizaciones locales se sometan a un proceso adaptativo que no puede predecirse con exactitud ni controlarse desde el exterior (p. 32).

Lo acontecido con las dos empresas contratistas que ya no hacen parte del sistema, es una muestra de cómo las situaciones que se pueden presentar en niveles inferiores a la administración, tienen repercusiones importantes en los planes de las autoridades que encabezan la jerarquía lineal expuesta, alterando el proceso de implementación del SITP. Igualmente, esta conducta se replica en las estrategias que desarrolló TransMilenio S. A. para remediar la salida de Coobús y Egobús, pues las 7 empresas restantes, al manifestar que no podían seguir prestando su apoyo para lidiar con el problema, fueron la razón por la cual se decidió retornar a los buses del TPC bajo un esquema provisional. 
Para Laurence J. O’Toole (2000), la implementación se puede resumir en la siguiente pregunta: “¿Qué sucede entre el establecimiento de la política y su impacto en el mundo de la acción?” (p. 273). La repuesta con base en el ejemplo del SITP Provisional es que todo puede ocurrir en esta etapa por más bienintencionadas y estructuradas que estén las decisiones de los policy-makers, pues la provisionalidad fue el tener que retornar a aquel viejo sistema obsoleto, para evitar que los cambios positivos del nuevo modelo de transporte del SITP se quedaran sin materializar, algo que en últimas puede sonar sumamente contradictorio.

\section{A MOdo DE CONCLUSIÓN}

El Sistema Integrado de Transporte Público de Bogotá, buscando ser una política que pretendía revolucionar la calidad del transporte, se estancó por fallos en su proceso de implementación bajo una lógica secuencial, la cual no va más allá de los mismos dilemas que surgen desde lo que se diseña en política pública y lo que realmente se ejecuta.

Teniendo en cuenta la naturaleza del SITP, fue una política pública compuesta por actores estatales y no estatales como la Secretaría Distrital de Movilidad, la empresa TransMilenio S. A., los contratistas ganadores de la etapa licitatoria y los transportadores tradicional del TPC en virtud del principio de democratización expuesto.

El modelo top-down presenta considerables falencias en cuanto a su contenido prescriptivo y racional, siendo un sesgo permanente que la autoridad pública debe considerar a forma de autocrítica, aún más teniendo en cuenta que quienes se dedicaron a investigar acerca de la etapa de implementación en políticas públicas, advirtieron de estos errores desde hace varias décadas.

Desde el modelo bottom-up, el concepto alternativo de burocracia, el enfoque hacia los grupos que no hacen parte de la elite decisoria y a la forma en cómo estos reaccionan a las disposiciones de estas autoridades, plantea alternativas que desde el punto de vista del análisis y la investigación del proceso de implementación de políticas públicas, pueden aportar a una visión más heurística de los problemas.

La actualidad del SITP es un reflejo del anterior análisis reflexivo, pues existe una iniciativa del Gobierno del alcalde Enrique Peńalosa para solucionar el problema de la provisionalidad 
del SITP, a la cual Apetrans se opone, pues argumenta que es algo inviable para sus economías internas debido incluso a la precariedad en la que se encuentran, pues denuncian haber sido afectados de forma gravosa en sus patrimonios tanto empresariales como personales, incluida su salud y relaciones personales.

La propuesta actual del Distrito, según Apetrans en su entrevista, consiste en un sistema temporal donde deben trabajar por 3 años, pintar los buses de azul (color característico de la modalidad "urbano"), cambiar el modo de recaudo, es decir, ponerles registradoras, garantizar más conductores disponibles, pagar el $8 \%$ al recaudador, el $5 \%$ a TransMilenio S. A. y conformar 5 operadoras que los agruparían para prestar el servicio.

La asociación de propietarios, señaló que se reclama desde que empezaron los problemas en la implementación del SITP, hasta la actualidad, el pago de las rentas dejadas de percibir por ellos a causa de su participación en Coobús S. A. S. y Egobús S. A. S., además de la prometida compra de sus buses, en donde enfatizan que no son simples vehículos, o chatarra como algunos afirman, sino que es un negocio, una empresa, es decir, es un conjunto de muchos elementos que les proporciona su sustento diario, por este motivo tiene un valor mayor a la luz de las leyes comerciales ${ }^{21}$.

Lo anterior demuestra que el panorama con el SITP se encuentra nublado y falto de soluciones, pues no existe ningún modo o forma concreta de mediación, donde ambas partes puedan ceder y acordar un mutuo beneficio, Apetrans reafirma que los dejen como están, es decir, bajo la figura provisional, o que les paguen lo debido y se retiran del transporte.

De igual manera, no hay claridad en las acciones por parte del Gobierno local; recientemente en noviembre de 2017, el alcalde de turno Enrique Peńalosa ha manifestado incluso la posibilidad de terminar con el SITP, argumentando los más de 650000 millones de pesos anuales que salen del presupuesto de la ciudad con destino a subsidiar el sistema, y los más de 8 billones de pesos en pérdidas debido a la crisis financiera según los voceros de las 7 empresas operadoras que siguen prestando el servicio.

21 Ver arts. 515 y 516 sobre la definición del establecimiento de comercio y sus elementos integrantes, Decreto 410 de 1971 Código de Comercio. 
El SITP permanece en estado de incertidumbre debido a estos errores en la fase de ejecución desde el punto de vista del estatuto que le dio origen; el problema de movilidad y transporte público en Bogotá sigue latente y a la espera de soluciones eficientes y eficaces.

\section{REFERENCIAS}

Acevedo, J. et al. (2009). El transporte como soporte al desarrollo de Colombia: una visión al 2040. Bogotá D. C., Colombia: Ediciones Uniandes.

Aguilar, C., y Lima, M. (2009). ¿Qué son y para qué sirven las políticas públicas? Contribuciones a las Ciencias Sociales. Septiembre 2009. Recuperado de www.eumed.net/rev/ cccss/05/aalf.htm

Alcaldía Mayor de Bogotá. (2015). Transición del TPC al SITP entra en su etapa final. Secretaría General de la Alcaldía de Bogotá Distrito Capital, comunicado digital del 31 de mayo de 2015. Recuperado de http://www.bogota.gov.co/article/ transición-del-tpc-al-sitp-entra-en-su-etapa-final

Anderson, J. (2015). Public Policymaking. Corpus Christi, TX: Texas. A\&M University. Cengage Learning.

Ardila, A. (2005). La olla a presión del transporte público en Bogotá. Revista de Ingeniería, (21), 54-67. Recuperado de http://www.redalyc.org/pdf/1210/121014218014.pdf

Asprilla, Y. y Rey, E. (2012). La implementación del Sistema Integrado de Transporte Público (SITP) de Bogotá y sus retos en el futuro. Tecnogestión, 9(1), 26-40. Recuperado de http://revistas.udistrital.edu.co/ojs/index.php/tecges/article/view/5649/7191

Ávila, D. (2011). Efectos clientelistas y corporativistas derivados de las normas de contratación estatal (tesis de maestría). Pontificia Universidad Javeriana, Bogotá D. C., Colombia.

Berman, P. (1978). The study of macro and micro implementation of social policy. Santa Mónica, CA: The Rand Papers Series.

Blanco, C. (2017). Condiciones de cohesión entre la comunidad andina (CAN) y la descentralización territorial colombiana. Revista Republicana, 23(2), 67-92. DOI: http:// dx.doi.org/10.21017/Rev.Repub.2017.v23.a31. Recuperado de http://ojs.urepublicana. edu.co/index.php/revistarepublicana/article/view/406/359 
Borda, S. (2015). Determinantes de la cooptación de la contratación pública en la administración de Samuel Moreno (tesis de pregrado). Universidad Colegio Mayor de Nuestra Señora del Rosario, Bogotá D. C., Colombia.

Botero, M., y Alegría, D. (2017). ¿La nueva tecnología en el sector transporte obstaculiza o fortalece el servicio público? Revista Verba Iuris, 12(37), 25-41. Recuperado de file://C:/Users/Usuario/Downloads/1022-Texto\%20del\%20art\%C3\%ADculo-15271-10-20170920.pdf

Calderón, D. (2016). Las políticas públicas: una construcción del valor público en la gobernabilidad. En D. Calderón (Comp.), Políticas públicas: retos y desafíos para la gobernabilidad (pp. 59-78). Bogotá D. C., Colombia: Universidad Santo Tomás, Ediciones USTA.

Cárdenas, J. (2005). Transición política y reforma constitucional en México. México D. F., México: Instituto de Investigaciones Jurídicas UNAM.

Castro, G. (2018). Una nueva propiedad intelectual para el siglo xxi. Focos, críticas y propuestas puntuales para una futura regulación de los bienes inmateriales. Revista IUSTA, 1(48), 19-41. DOI: https://doi.org/10.15332/s1900-0448.2018.0048.01. Recuperado de http://revistas.usta.edu.co/index.php/iusta/article/view/4085/3880

Decreto 319. (15 de agosto de 2006). Adopción del Plan Maestro de Movilidad para Bogotá Distrito Capital. Alcaldía Mayor de Bogotá. Recuperado de http://www.alcaldiabogota. gov.co/sisjur/normas/Norma1.jsp?i=21066

Decreto 309. (23 de julio de 2009). Adopción del Sistema Integrado de Transporte Público para Bogotá D. C. Alcaldía Mayor de Bogotá. Recuperado de http://www.alcaldiabogota.gov.co/sisjur/normas/Norma1.jsp?i=36852\#8

Dinero. (2010). Transporte en Bogotá: ¿Quiénes están detrás? Dinero. Recuperado de http://www.dinero.com/edicion-impresa/negocios/articulo/transporte-bogota-quienesestan-detras $/ 91263$

Duarte, C. (2015). Movilidad urbana: ¿cómo definir un sistema de transporte público colectivo (SITP), en Bogotá, D. C. Colombia? Cuadernos Latinoamericanos de Administración, 11(20), 71-82. Recuperado de http://www.redalyc.org/pdf/4096/409640743007.pdf

Elmore, R. (1978). Organizational Models of Social Program Implementation. Public Policy, 26(2), 185-228. 
Estévez, A., y Esper, S. (2005). Revisando el modelo secuencial de políticas públicas. Revista del Instituto AFIP. IR05, 72-90.

González, J. (2017). Una reflexión sobre el paradigma de la integralidad en el transporte público de Bogotá. Revista Via Inveniendi et Iudicandi, 12(1), 149-178. Recuperado de http://revistas.usantotomas.edu.co/index.php/viei/article/view/3559/3447

Hill, M., y Hupe, P. (2002). Implementing public policy: governance in theory and in practice. Londres, Reino Unido: SAGE Publications.

Jones, C. (1970). An introduction to the study of public policy. Belmont, CA: Duxbury Press.

Llano, J. V., Rengifo, R. A., y Rojas, L. M. (2018). Estado cosmopolita en América Latina. Revista IUSTA, 1(48), 97-117. DOI: https://doi.org/10.15332/s1900-0448.2018.0048.04. Recuperado de http://revistas.usta.edu.co/index.php/iusta/article/view/4088/3883

Lindblom, C. (1959). The science of 'muddling through'. Administration Review, 19(2), 79-88.

Lipsky, M. (2010). Street-Level Bureaucracy. Dilemmas of the individual in public services. NY: Russell Sage Foundation.

Lleras, G. C. (2005). TransMilenio y el transporte colectivo tradicional, una relación incierta. Revista de Ingeniería, (21), 83-93. Recuperado de http://www.scielo.org.co/scielo. php?script=sci_arttext\&pid=S0121-49932005000100009\&lng=es\&nrm=iso\&tlng=es

Matland, R. (1995). Synthesizing the Implementation Literature: The Ambiguity-Conflict Model of Policy Implemenation. Journal of Public Administration Research and Theory, 4(2), 145-174. Recuperado de http://citeseerx.ist.psu.edu/viewdoc/download?doi=10.1. 1.607.9799\&rep=rep $1 \&$ type $=$ pdf

Meny, Y., y Thoenig, J. (1992). Politicas públicas (Francisco Morata, trad.). Barcelona, España: Editorial Ariel S. A. (Obra original publicada en 1989).

Morata, F. (1990). Políticas públicas y relaciones intergubernamentales. Documentación administrativa, (224-225), 153-167. Recuperado de https://s3.amazonaws.com/academia.edu.documents/30828344/RIG_Morata.pdf?AWSAccessKeyId=AKIAIWOWYY GZ2Y53UL3A\&Expires= $1528342143 \&$ Signature $=u 4 \% 2 \mathrm{BtOZtz0w} 7 \mathrm{tgfcqH} \% 2 \mathrm{Fa} 2 \mathrm{~b}$ WlyXw4\%3D\&response-content-disposition=inline\%3B\%20filename\%3DPoliticas_ publicas_y_relaciones_intergub.pdf 
O’Donnell, G., y Schmitter, P. (2001). Transiciones desde un gobierno autoritario. Conclusiones tentativas sobre las democracias inciertas. Buenos Aires, Argentina: Paidós Estado y Sociedad.

O’Toole, L. J. (2000). Research on policy implementation: Assessment and prospects. Journal of public administration research and theory, 10(2), 263-288. Recuperado de https:// ris.utwente.nl/ws/portalfiles/portal/7053582

Parsons, W. (2007). Politicas públicas: una introducción a la teoría y la práctica del análisis de politicas públicas (Atenea Acevedo, trad.). México D. F., México: Facultad Latinoamericana de Ciencias Sociales FLACSO. (Obra original publicada en 1995).

Paudel, N. (2009). A Critical Account of Policy Implementation Theories: Status and Reconsideration. Nepalese Journal of Public Policy and Governance. 25(2), 36-54. Recuperado de http://sjpg.pactu.edu.np/system/files/journal/articles/3-narendra-paudel-acritical-account-of-policy-implementation-theories.pdf

Peña, V. (2011). Apuntes teóricos sobre la implementación de políticas públicas. Revista Electrónica del Centro de Estudios en Administración Pública FCPyS UNAM, (9), 1-14. Recuperado de file://C:/Users/Usuario/Downloads/58470-168917-1-PB.pdf

Pressman, J., y Wildavsky, A. (1984). Implementation: How great expectations in Washington are dashed in Oakland. Berkeley, CA: University of California Press.

Revuelta, B. (2007). La implementación de políticas públicas. Dikaion, 21(16), 135-156. Recuperado de http://www.redalyc.org/pdf/720/72001610.pdf

Roth, A. (2002). Politicas públicas: formulación, implementación y evaluación. Bogotá D. C., Colombia: Ediciones Aurora.

Roth, A. (2010). Las políticas públicas y sus principales enfoques analíticos. En A. Roth (Ed.), Enfoques para el análisis de politicas públicas (pp. 17-56). Bogotá D. C., Colombia: Universidad Nacional de Colombia.

Sabatier, P., y Mazmanian, D. (1980). The implementation of public policy: A framework of analysis. Policy studies journal, 8(4), 538-560. Recuperado de https://onlinelibrary.wiley. com/doi/pdf/10.1111/j.1541-0072.1980.tb01266.x

Secretaría Distrital de Movilidad. (8 de julio de 2015). Permiso de operación especial y transitorio para operar las rutas provisionales del Sistema Integrado de Transporte Público 
del Distrito Capital “SITP”. [Resolución 518 de 2015]. Recuperado de http://www. alcaldiabogota.gov.co/sisjur/normas/Norma1.jsp?i=62553

Sen, A. (1998). Las teorías del desarrollo a principios del siglo xxi. En L. Emmerij y J. Núńez (Eds.), El desarrollo económico y social en los umbrales del siglo XXI (pp. 73-100). Washington D. C.: Banco Interamericano de Desarrollo.

Stahl, K. (1994). Política social en América Latina. La privatización de la crisis. Nueva Sociedad, 131, 48-71. Recuperado de file://C:/Users/Usuario/Downloads/Stahl\%20(1).pdf

Subirats, J. (1992). Análisis de politicas públicas y eficacia de la administración. Madrid, España: Ministerio para las Administraciones Públicas.

Superintendencia de Puertos y Transporte. (s. f.). Coobús y Egobús aprobaron sus planes de salvamento. Autor. Recuperado de http://www.supertransporte.gov.co/index.php/2uncategorised/265-coobus-y-egobus-aprobaron-sus-planes-de-salvamento.html

Superintendencia de Sociedades. (13 de septiembre de 2016). Supersociedades decreta liquidación judicial de Coobús S. A. S. y Egobús S. A. S. Autor. Recuperado de http://www.supersociedades.gov.co/noticias/Paginas/2016/SuperSociedades-decretaliquidaci\%C3\%B3n-judicial-de-Coobus-SAS-y-Egobus-SAS.aspx

Trujillo, I. A. (2018). Las instituciones en la administración financiera de las misiones diplomáticas de Colombia en el exterior. Revista IUSTA, 1(48), 145-178. DOI: https://doi.org/10. 15332/s1900-0448.2018.0048.06. Recuperado de http://revistas.usta.edu.co/index. php/iusta/article/view/4102/3906

Urazán, C., y Velandia, E. (2012). Consideraciones sobre el esquema del Sistema Integrado de Transporte Público para Bogotá. Épsilon (18), 105-121. Recuperado de http://oaji. net/articles/2015/2065-1432506524.pdf

Van Meter, D. S., y Van Horn, C. E. (1975). The policy implementation process: A conceptual framework. Administration \& Society, 6(4), 445-488. 